\title{
Machine Learning-based Prognostics of Fatigue Crack Growth in Notch Pre-cracked Aluminum 7075-T6 Rivet Hole
}

\author{
Robert Haynes ${ }^{1}$, Ghanashyam Joshi ${ }^{2}$, and Natasha Bradley ${ }^{3}$ \\ ${ }^{1}$ US Army Research Laboratory, Aberdeen Proving Ground, MD, 21005, USA \\ robert.a.haynes43.civ@mail.mil \\ ${ }^{2}$ Southern University and A\&M College, Baton Rouge, LA, 70813, USA \\ ghanashyam_joshi@subr.edu \\ ${ }^{3}$ US Army Research Laboratory, Aberdeen Proving Ground, MD, 21005, USA \\ natasha.c.epps-bradley.civ@mail.mil
}

\begin{abstract}
Constant stress amplitude fatigue tests were conducted on the notch pre-cracked Aluminum 7075-T6 rivet hole dog-bone coupons. Monitoring of visible surface crack length by special surface engraving using digital microscope images and by ultrasonic sensors signals was carried out to yield fatigue crack length measurements in relation to number of fatigue cycles applied. The experimental results provide ultrasonic sensor validation for fatigue crack length measurements. Fracto-graphic examination of failed fatigue surfaces has provided further confirmation of notch pre-crack length, crack initiation process, and crack growth marker bands. These experimental inputs were used in NASGRO and AFGROW software fatigue crack growth simulations. The simulation results did not match the crack initiation fatigue life measured by experiments. However, there was good agreement with crack growth simulations of larger cracks. Hence, we plan to develop a machine learning application that will learn the fatigue crack initiation and crack growth processes from data obtained from our own experiments and other fatigue data available from AFGROW databases. Nonlinear AutoRegressive models with eXogenous input (NARX) artificial neural network were used to predict crack growth longer than 5.0-mm. Particle filtering modeling with Bayesian updating was applied to these experimental data for prognostics of fatigue crack growth. A concept design and preliminary implementation results will be presented.
\end{abstract}

Robert Haynes et al. This is an open-access article distributed under the terms of the Creative Commons Attribution 3.0 United States License, which permits unrestricted use, distribution, and reproduction in any medium, provided the original author and source are credited.

\section{BACKGROUND AND MOTIVATION}

Accurate estimation of fatigue crack initiation cycles and growth rates are crucial to safe and efficient operation of air vehicles. Fatigue crack growth simulation software packages such as NASGRO (NASGRO) are commonly used for estimation of fatigue crack parameters. However, fatigue life and crack length estimates have been found to be wrong by a factor of as much as tenfold (Haile, 2012 and Vasudevan, 2016). The main contributing factor of such large errors is the use of equivalent initial flaw size (EIFS) (Newman, 2016). EIFS is commonly calculated through back extrapolation from a long fatigue crack using the Paris crack growth law (Paris, 1963). The EIFS has been reported as a material property (Johnson, 2010); however, the authors argue that it is in fact application dependent. The proposed effort uses a measurable notch pre-crack introduced on the rivet hole of the dog-bone shaped Aluminum 7075-T6 coupon.

The motivation for this effort is the U.S. Army aviation community's desire to achieve a "zero-maintenance aircraft." To that end, the U.S. Army Research Laboratory is conducting research into damage precursors (Weiss, 2014). When linked with crack-growth models, the ability to predict damage initiation through precursor detection will enable prognostics that can provide a risk-based estimate of the remaining useful life of a structure. Zio (2010) demonstrated the ability to predict crack growth in metal using particle filtering. This work aims at addressing the challenges of this approach by (1) using structural health monitoring techniques to detect when crack initiation occurs and (2) linking crack initiation to crack-growth models to predict when failure will occur using experimental data. 


\section{APPROACH}

Twenty seven coupons have been tested, and the data collected includes the number of cycles, crack length, and structural health monitoring system sensor data. The coupons used for crack growth are Al 7075-T6 dog-bone shaped, which have a nominal thickness of $1.6 \mathrm{~mm}(0.063$ inch), ultimate tensile strength of $715 \mathrm{MPa}$ (75 ksi), yield stress of $482 \mathrm{MPa}$ (69.9 ksi), and modulus of elasticity of 71.7 GPa $(10,400 \mathrm{ksi})$. Figure 1 (Iglesias, 2015) shows the dimensions of the fatigue test coupon, which was machined with a rivet size hole and a notch pre-crack. In order to accelerate crack growth, so that fatigue failure would occur in approximately 50,000 cycles, a notch cut was applied to the right of the hole in a direction perpendicular to the length of the specimen. The notch cut was administered by a jewelers saw blade or wire EDM process $0.178 \mathrm{~mm}$ (7 mil) thick. The crack propagation area, shown in Figure 1, is where the crack is expected to propagate due to the notch cut.

A neural network and Bayesian particle filter based machine learning application is presented to predict crack initiation life and crack growth life for constant amplitude stress cycles applied to notch pre-cracked Aluminum 7075-T6 rivet hole coupons. The crack initiation life covers over $60 \%$ of the total fatigue life. The unique feature of the machine learning application is that it will cover the entire fatigue life cycle.

\subsection{Fatigue Test Parameters}

Coupons were subjected to constant stress amplitude fatigue test on a servo-hydraulic MTS test machine. A cyclic load cycle with maximum load of $5 \mathrm{kN}(1,124 \mathrm{lb})$ and a minimum load of $500 \mathrm{~N}$ (112.4 lb). This was done to prevent debonding within the adhesive of the structural health monitoring system. The nominal geometry shown in Figure 1 , gave a nominal far field cyclic stress ranging from $11 \mathrm{MPa}$ to $114 \mathrm{MPa}$ (1.66 ksi to $16.57 \mathrm{ksi}$ ), with stress ratio $\mathrm{R}=0.1$, during each of the fatigue tests. The fatigue test was halted every 500 cycles to capture a digital photograph of the coupon surface and to collect ultrasonic pitch catch signals from the structural health monitoring system. The special surface engraving enabled an automated estimation of the surface crack length, which was recorded every 500 cycles.

\subsection{Structural Health Monitoring System}

The Structural Health Monitoring (SHM) system used consisted of an Acellent Technologies, Inc. SMART layer System with piezoelectric transducers and ScanGenie-II hardware. The hardware produces a hamming windowed pulse in one transducer, which in turn creates a surface wave that travels through the specimen, interacts with the boundary conditions (including damage), and is detected in another transducer. The transducers were configured in pitch-catch design such that three transducers, numbered $1-3$, on one side of the hole act as actuators to generate the pulse and three transducers, numbered 4-6, on the other side of the hole act as sensors to detect the pulse; see Figure 2 (Iglesias, 2015).

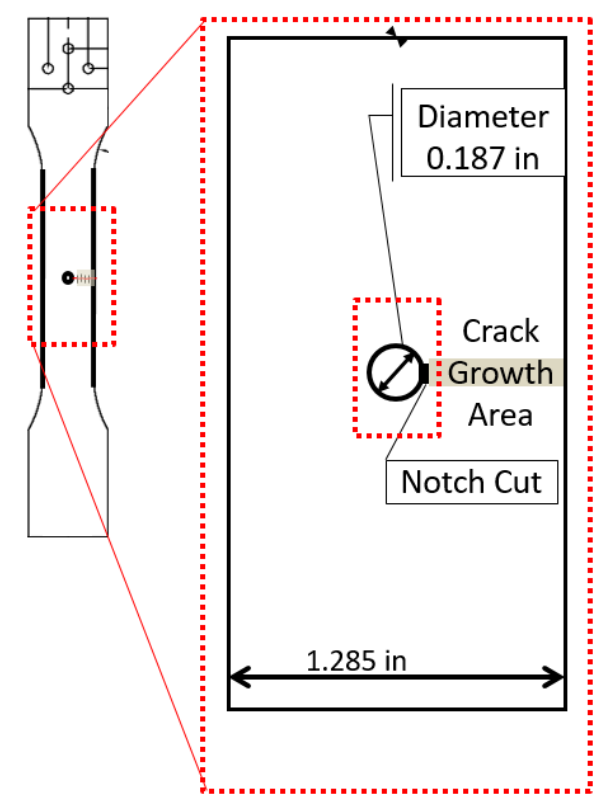

Figure 1. Fatigue test coupon geometry.

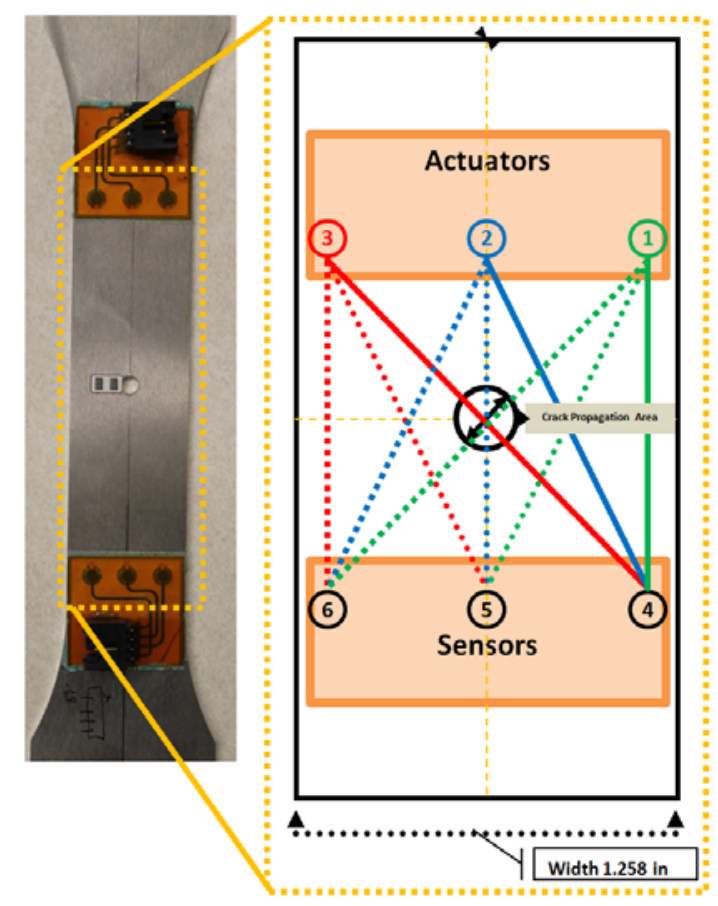

Figure 2. Structural health monitoring system layout. 
Reducing the raw sensor data to a damage index is necessary to establish a relationship with crack length. One commonly used damage index uses Pearson's correlation coefficient to relate the sensed signal from a damaged state to a baseline sensed signal (Vehorn, 2013).

$$
\text { Damage Index }(D I)=1-\rho_{x y}
$$

where,

$$
\rho_{x y}=\frac{E\left[\left(x-\mu_{x}\right)\left(y-\mu_{y}\right)\right]}{\sqrt{\operatorname{Var}(x)} \sqrt{\operatorname{Var}(y)}}
$$

$x$ and $y$ are the independent and dependent variables, respectively, $\mu$ is the mean, $E$ is the expected value, and $\operatorname{Var}()$ is the variance.

\section{SAMPLE RESULTS}

Sample results for one specimen are provided in Figure 3 (Iglesias, 2015). In this case, the specimen failed after slightly more than 50,000 cycles. The black dotted line indicates actual crack size as measured optically. The colored lines indicate damage index values for the nine unique paths from each actuator to each sensor. Paths containing actuator 3 and sensor 6 do not exhibit high sensitivity; this is intuitive because actuator 3 and sensor 6 are on the opposite side of the hole from the crack. Paths containing sensor 5 appear to be the most sensitive to damage; however, their sensitivity decreases after about 40,000 cycles. Ongoing efforts are aimed at understanding this phenomenon. Path 1-4 is the only path that exhibits a monotonic trend with fatigue life; therefore, it will be used to create a calibration curve for linking damage index to crack size.

\subsection{Experimental Results}

The results of fatigue test experiments are summarized in Table 1, grouped by initial notch size. For brevity only the number of cycles (in thousands) required to achieve crack lengths, $C$, of 0.04 in $(1 \mathrm{~mm}), 0.10$ in $(2.5 \mathrm{~mm}), 0.20$ in (5 $\mathrm{mm}), 0.40$ in $(10 \mathrm{~mm})$, and 0.435 in $(11 \mathrm{~mm})$ are presented. The crack initiation cycles required to observe 0.04 in $(1 \mathrm{~mm})$ crack ranged from 7,500 to 22,000 cycles. The fatigue life ranged from 22,500 to 52,000 cycles. It can be observed from Table 1 that the fatigue cycles required to initiate and grow the cracks to failure generally decrease with the increase in notch length; however, there are outliers represented by fatigue performance of three coupons shown by dotted lines in Figure 4 which have higher notch lengths and yet they show higher fatigue life for crack initiation and fatigue failure. Some other factors such as favorable notch tip geometry and absence of defects must be at play governing the delayed fatigue crack initiation and growth. Moreover, the NASGRO and AFGROW simulations required though thickness crack growth governed by linear elastic fracture mechanics (LEFM). Some of these requirements may be violated in experiments and in practice. The crack appeared to inititate from a corner and grow through through the thickness before propagating to the edge. Hence, further fractographic examination and investigation of notch effects will be carried out in future experimental efforts.

\subsection{Fatigue Crack Growth Simulation And Analysis}

This effort consists of simulation and analysis of fatigue crack growth using the NASGRO and AFGROW software applications. The effort consists of four parts: (1) determining the Equivalent Initial Flaw Size (EIFS) for NASGRO and initial crack size for AFGROW, (2) calculating the stress intensity factor from the notch, (3) predicting crack growth using NASGRO and AFGROW, and (4) integrating structural health monitoring with NASGRO and AFGROW predictions to provide more accurate predictions of future crack size. Out of these, only the items 1 and 3 have been completed and emphasized in this work.

The first part involves determining the EIFS. This is done by taking measurements of crack size during the crack growth regime and back-extrapolating to zero cycles using the Paris Law [5] or one of its derivatives. The EIFS forms the basis for predicting the number of cycles to crack initiation or critical crack formation. NASA recommends initial flaw sizes range from 0.005 in to $0.25 \mathrm{in}$, depending on manufacturing and inspection methods. For our simulation work, the EIFS predictions were carried out in NASGRO using selected fatigue life to failure cycles shown in Table 2, column 1. The EIFS estimates presented in Table 2 are used for NASGRO NASFLA module using TC13 geometry. The NASGRO simulation results are summarized in Table 3.

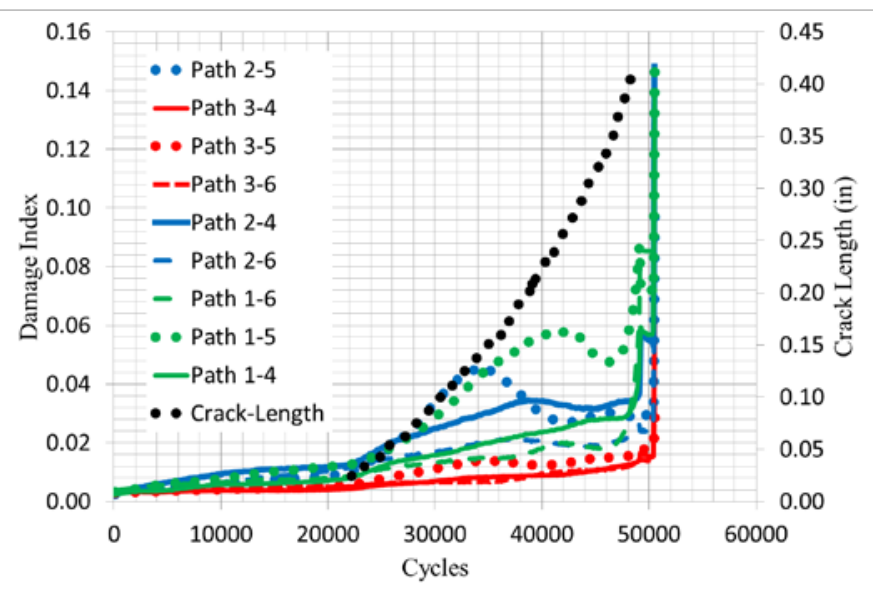

Figure 3. Damage indices and measured crack length. 


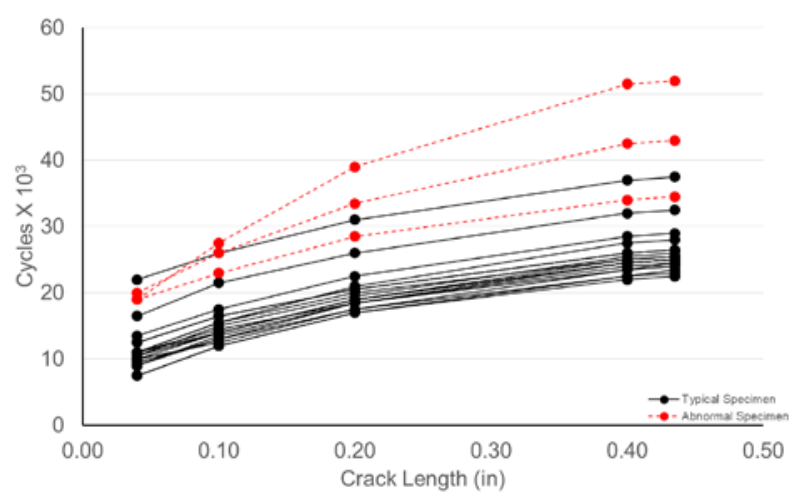

Figure 4: Cycles (Thousands) versus crack length, C

Table 1 . Thousands of cycles to reach crack lengths, $C$, of a given size, grouped by approximate notch size.

\begin{tabular}{lcccc}
\hline $\mathrm{C}=0.04$ & $\mathrm{C}=0.10$ & $\mathrm{C}=0.20$ & $\mathrm{C}=0.40$ & $\mathrm{C}=0.44$ \\
\hline $0.003 \mathrm{IN}$ & $\mathrm{NOTCH}$ & & & \\
22.0 & 26.0 & 31.0 & 37.0 & 37.5 \\
16.5 & 21.5 & 26.0 & 32.0 & 32.5 \\
$0.007 \mathrm{IN}$ & $\mathrm{NOTCH}$ & & & \\
10.5 & 14.0 & 19.0 & 24.5 & 25.0 \\
19.0 & 23.0 & 28.5 & 34.0 & 34.5 \\
10.5 & 15.0 & 19.5 & 25.5 & 26.0 \\
10.5 & 14.0 & 19.0 & 24.5 & 25.0 \\
10.0 & 14.0 & 19.0 & 24.5 & 25.0 \\
$0.008 \mathrm{IN}$ & NOTCH & & & \\
9.50 & 13.0 & 17.5 & 23.5 & 24.0 \\
11.0 & 15.5 & 20.0 & 25.0 & 25.5 \\
11.0 & 15.5 & 21.0 & 27.5 & 28.0 \\
11.0 & 14.5 & 18.5 & 23.5 & 24.5 \\
12.5 & 16.5 & 20.5 & 26.0 & 26.5 \\
13.5 & 17.5 & 22.5 & 28.5 & 29.0 \\
8.0 & 12.0 & 16.5 & 22.5 & 23.0 \\
0.010 IN NOTCH & & & \\
11.0 & 14.0 & 19.0 & 25.0 & 25.5 \\
7.50 & 12.0 & 17.0 & 22.0 & 22.5 \\
7.50 & 12.0 & 17.0 & 22.5 & 23.5 \\
19.0 & 27.5 & 39.0 & 51.5 & 52.0 \\
10.0 & 12.5 & 17.5 & 22.5 & 23.0 \\
0.012 IN NOTCH & & & \\
20.0 & 26.0 & 33.5 & 42.5 & 43.0 \\
9.00 & 13.5 & 18.5 & 24.0 & 24.5 \\
9.00 & 13.0 & 18.5 & 24.5 & 25.0 \\
\hline
\end{tabular}

Table 2. NASGRO predictions of number of cycles to failure for various initial flaw sizes.

\begin{tabular}{cc}
\hline Life (Cycles) & Initial flaw (in) \\
\hline 23,000 & 0.0526 \\
25,000 & 0.0404 \\
30,000 & 0.0159 \\
35,000 & 0.0046 \\
40,000 & 0.0016 \\
43,000 & 0.0010 \\
51,000 & 0.0004 \\
\hline
\end{tabular}

The initial crack size input for AFGROW simulations was (Notch size $+0.5^{*}$ Thickness). The experimentally observed crack length is AFGROW simulation crack length minus the notch length. This correction was applied to the AFGROW simulation output crack lengths. The number of cycles required to make an initial 0.0315 in (half thickness) was taken from the corresponding experimental observations. These cycles were then added to the AFGROW simulation results starting from this initial 0.0315 in observed crack length. The results of AFGROW simulation using NASGRO equation and Harter-T method, provided in the first and second rows of each entry, respectively, are shown in Table 4. The first column shows the initial crack size input provided to AFGROW software for running the simulation. Subsequent columns of Table 4 show the observed crack lengths $C$, and corresponding thousands of cycles required to reach those crack lengths, after applying the correction adjustment described above. The results presented in Table 3 and Table 4 can be compared to experimental data presented in Table 1, to determine under what conditions the predictions are valid, and if not, how inaccurate they are.

Table 3. NASGRO predictions of thousands of cycles to reach selected crack lengths.

\begin{tabular}{cccccc}
\hline $\begin{array}{c}\text { Initial } \\
\text { flaw (in) }\end{array}$ & $\mathrm{c}=0.04$ & $\mathrm{c}=0.10$ & $\mathrm{c}=0.20$ & $\mathrm{c}=0.40$ & $\mathrm{c}=0.44$ \\
\hline 0.0526 & - & 6.32 & 15.1 & 22.6 & 22.9 \\
0.0404 & - & 8.45 & 17.2 & 24.7 & 25.1 \\
0.0159 & 4.92 & 13.4 & 22.1 & 29.6 & 30.0 \\
0.0046 & 9.91 & 18.4 & 27.1 & 34.6 & 35.0 \\
0.0016 & 14.9 & 23.3 & 32.1 & 39.6 & 40.0 \\
0.0010 & 17.9 & 26.3 & 35.1 & 42.6 & 43.0 \\
0.0004 & 26.0 & 34.4 & 43.2 & 50.6 & 51.0 \\
\hline
\end{tabular}


Table 4. AFGROW predictions of thousands of cycles to reach selected observed crack lengths $\mathrm{C}$; first line is NASGRO equation, second line is Harter-T method.

\begin{tabular}{cccccc}
\hline $\begin{array}{c}\text { Initial } \\
\text { Crack } \\
\begin{array}{c}\text { Size } \\
\text { Input } \\
\text { (in) }\end{array}\end{array}$ & $\mathrm{C}=0.04$ & $\mathrm{C}=0.10$ & $\mathrm{C}=0.20$ & $\mathrm{C}=0.40$ & $\mathrm{C}=0.44$ \\
\hline 0.0411 & 8.50 & 17.5 & 28.0 & 37.5 & 38.0 \\
0.0411 & 8.00 & 14.0 & 21.0 & 27.0 & 27.5 \\
& & & & & \\
0.0383 & 11.0 & 20.0 & 30.5 & 40.5 & 41.0 \\
0.0383 & 10.5 & 16.5 & 23.5 & 30.0 & 30.5 \\
& & & & & \\
0.0392 & 11.5 & 21.5 & 31.0 & 41.0 & 41.5 \\
0.0392 & 11.0 & 17.0 & 24.0 & 30.5 & 31.0 \\
& & & & & \\
0.0434 & 19.5 & 29.0 & 39.0 & 48.5 & 49.0 \\
0.0434 & 19.5 & 25.5 & 32.5 & 38.5 & 38.7 \\
& & & & & \\
0.0341 & 23.0 & 32.0 & 43.0 & 53.0 & 54.0 \\
0.0341 & 22.5 & 28.5 & 36.0 & 42.5 & 43.0 \\
\hline
\end{tabular}

NASGRO and AFGROW both offer crack initiation or crack formation capabilities; however, data and inputs needed for reasonably accurate estimates are currently not available. Hence, both packages were unable to provide estimates that match with experimentally recorded data. Figures 4 through 7 provide graphical comparison of NASGRO, and AFGROW simulation results to the experimentally recorded fatigue cycle counts required to achieve selected crack lengths tabulated in Tables 1, 3, and 4. Significant mismatch between simulation results and experimental results is evident for crack initiation and growth up to about 0.2 in $(5.1 \mathrm{~mm})$ crack size. It should be noted that about $60 \%$ fatigue life is expended in crack initiation and more that $80 \%$ fatigue life is exhausted by the time fatigue crack grows to 0.2 in $(5.1 \mathrm{~mm})$. This motivates additional future work to gain accurate simulation results matching the experimental and eventually practical fatigue scenarios.

The NASGRO results match more closely to experimentally measured data presented in Table 1 , particularly towards the end of fatigue life. Using AFGROW, the Harter- $T$ method results are closer match with experimental results than the NASGRO equation, albeit with significant degree of mismatch for smaller cracks.

For smaller fatigue life cycles, 23,000 and 25,000, AFGROW did not produce satisfactory simulation results using the recommended initial crack size inputs; however, using higher values of initial flaw sizes of 0.0526 in and 0.0404 in simulated those fatigue life estimates, shown in Table 2. Since, an estimate of cycles to achieve crack lengths of 0.04 in cannot be made from initial flaw sizes greater than 0.04 and are omitted from the table. The results of NASGRO simulations, for these lower fatigue life cases, in comparison with experimental results are shown in Figure 8 and 9. These results show greater mismatch between simulation and experimental results from crack initiation to about 0.4 in (10 $\mathrm{mm}$ ) crack length. The simulation results show that for given number of cycles the crack length estimate is much higher than that experimentally observed. This motivates further investigation of crack initiation, closure effects, and deviations from LEFM models.

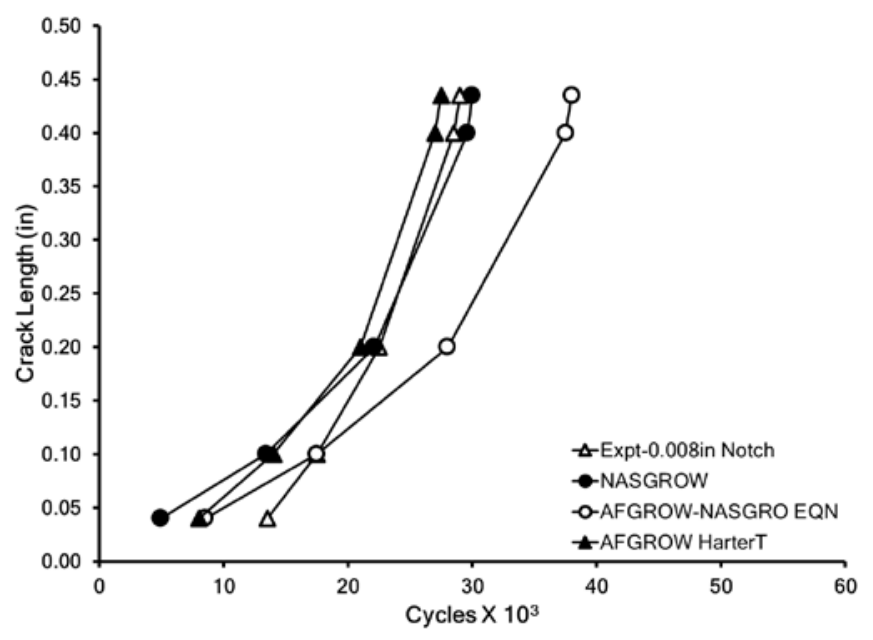

Figure 4: Comparison 1 of AFGROW, NASGROW results with Experimental results

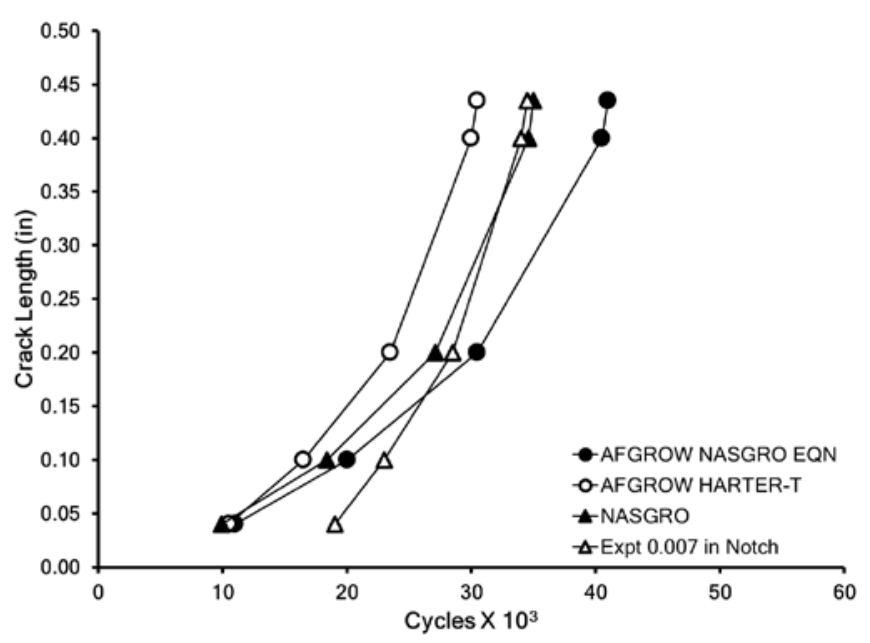

Figure 5: Comparison 2 of AFGROW, NASGROW results with Experimental results 


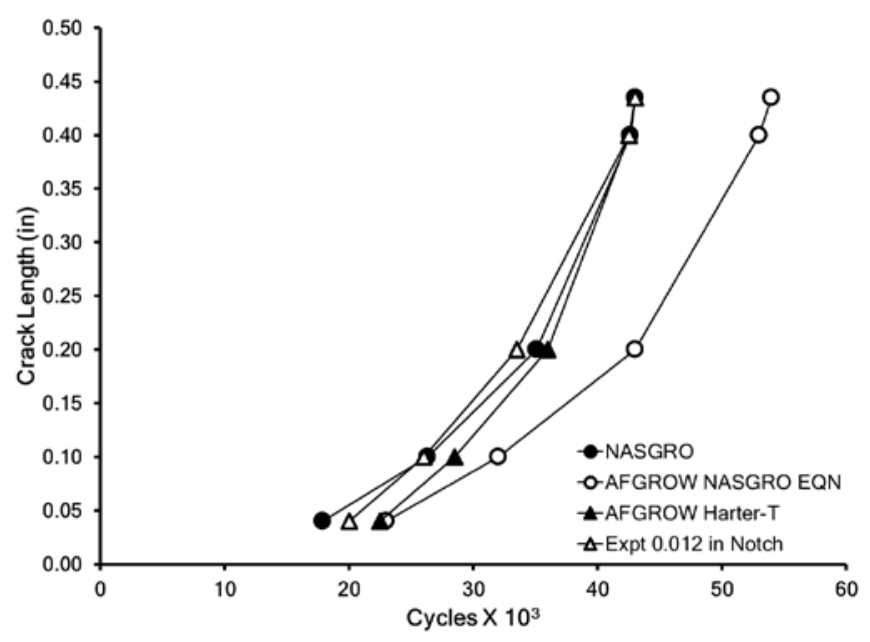

Figure 6: Comparison 3 of AFGROW, NASGROW results with Experimental results

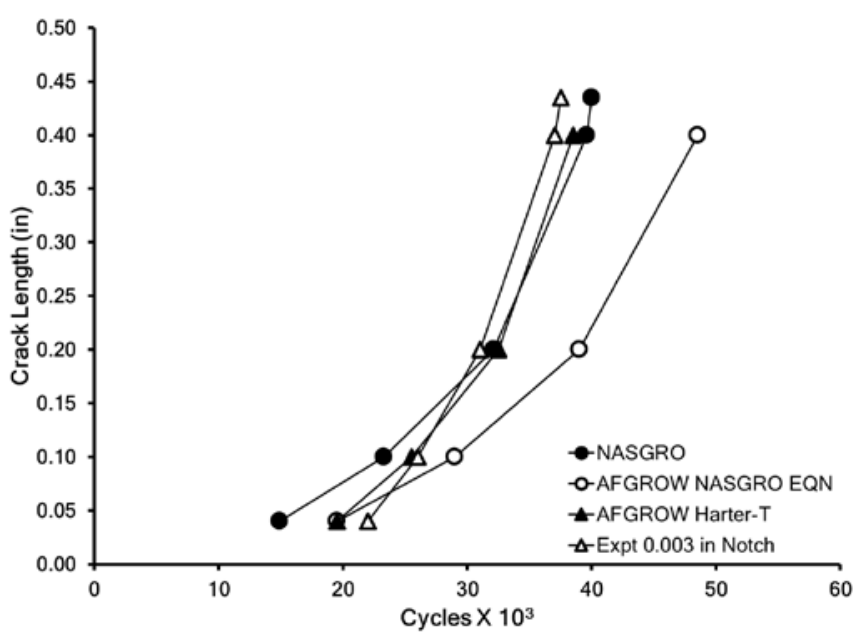

Figure 7: Comparison 4 of AFGROW, NASGROW results with Experimental results

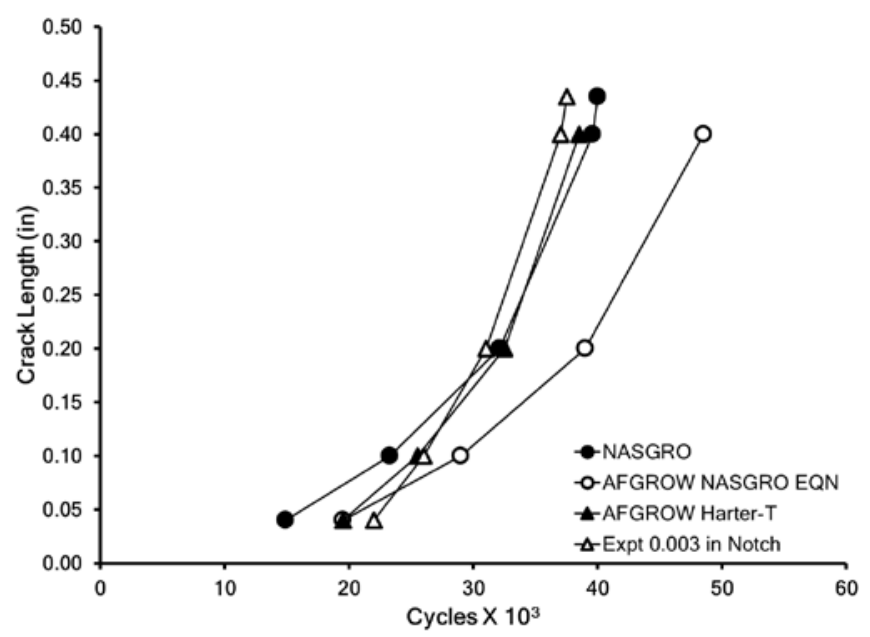

Figure 8: Comparison 1 of NASGRO results with Experimental results

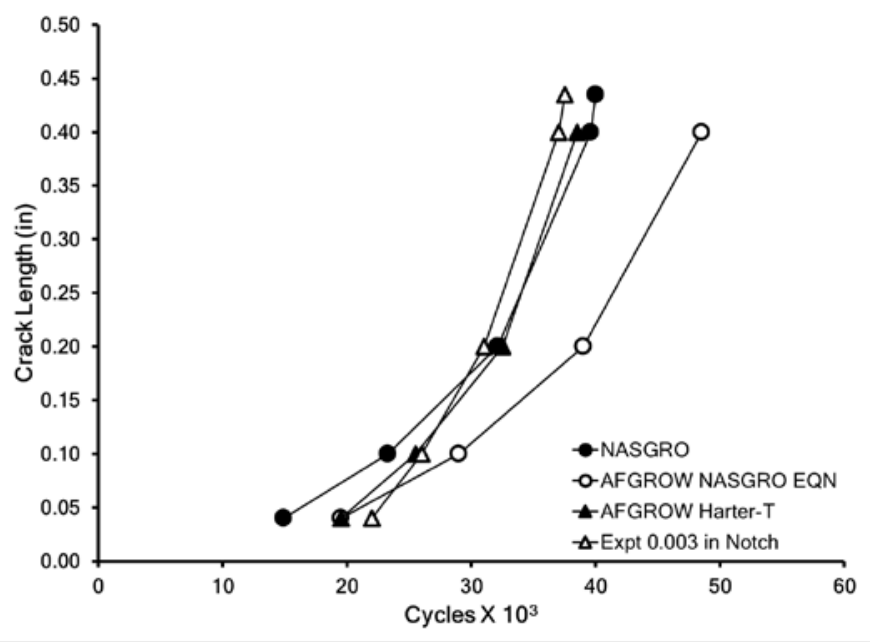

Figure 9: Comparison 2 of NASGRO results with Experimental results

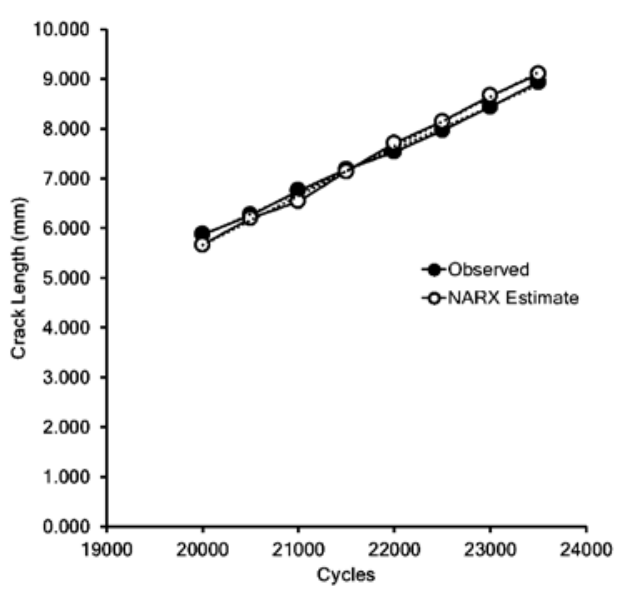

Figure 10: NARX modeling of crack growth

\section{NARX MODELING OF CRACK GROWTH}

Nonlinear AutoRegressive models with eXogenous input (NARX) artificial neural network were fitted to crack lengths less than $5.0 \mathrm{~mm}$. These were then used to predict crack growth longer than $5.0-\mathrm{mm}$. The crack length estimates are both shorter and longer than the actual experimentally measured crack lengths, within a 3\% error margin. The results are shown in Figure 10.

The following equation defines the proposed NARX model:

$$
y(t)=f\left(y_{(t-1)}, y_{(t-2)} \ldots, y_{(t-n y)}, u_{(t-1)}, u_{(t-2)}, u_{(t-n u)}\right)
$$

where $f$ is a non-linear function which will be approximated by multi-layer perceptron (MLP) NARX neural network, $y(t)$ and $u(t)$ are the output crack lengths and the input cycles, respectively, and $n_{y}$ is the output delays and $n_{u}$ are the input delays. Equation 3, represents the non-linear NARX model 
fitted to the output and input signals. Output $y(t)$ is nonlinearly auto-regressed on prior values of the output crack lengths and prior values of the number of fatigue cycles corresponding to those crack lengths. The technical capabilities of NARX models and their applications are described in literature (H. Siegelmann et al, 1997).

The NARX model was implemented with a feedforward neural network model in Matlab code. The model can be used to find an approximate value of non-linear function $f$ fitted to fatigue crack length data by NARX neural network. Scaled conjugate gradient backpropagation was used for training the NARX neural network. Two input delays $\left(n_{u}\right)$ and two output feedback delays $\left(n_{y}\right)$ were used. The $90 \%$ of fitted crack length data was used for training of NARX neural network, and 5\% each were used for validation and testing. The network had 10 hidden layers, a learning rate of 70 and had 9 neurons per layer with 40 epochs.

\section{Particle Filtering Results}

MatlLab-based implementation of fatigue crack growth particle filtering software (D. An, et al, 2013) was adopted for our experimental data. More in-depth applications of PF methods to fatigue crack growth are available in technical papers (E. Zio and G. Peloni, 2010). The applied Matlab code consists of problem definition, prognostics using PF, and post-processing for output of results. Initial crack size, in meters, was set to normal distribution $\sim N\left[0.002,\left(5.0 \times 10^{-4}\right)^{2}\right]$. Paris model for long fatigue crack length $\left(a_{k}\right)$ was assumed to be applicable to the experimentally measured fatigue crack growth data, $z_{k}$. The Paris model in state transition function form is as follows:

$$
a_{k}=C_{k}\left(1.2 \Delta \sigma \sqrt{\pi a_{k-1}}\right)^{m_{k}} d N+a_{k-1}
$$

The model parameters, $C_{k}$ and $m_{k}$, and $a_{k}$ are estimated using $z_{k}$ at every 500 cycles under constant stress amplitude of $\Delta \sigma$ $=114 \mathrm{MPa}$; the factor of 1.2 is used to account for the notched rivet hole coupon geometry. Initially, true crack sizes are generated using $m_{k}=3.4$ and $C_{k}=1.03 \times 10^{-10}$. Initial lognormal distribution for Paris law constant $C$ was assumed to be $\ln \left(C_{0}\right) \sim \mathrm{N}\left[-23,1.12^{2}\right]$. Initial Paris law exponent $m$ distribution was set to $\sim \mathrm{N}\left[3.4,0.2^{2}\right]$. The measurement noise was set as $\sim N[0.0001,0]$. Bayesian updating of posterior estimates using normal distribution likelihood function of priors was applied. The critical crack size and maximum stress amplitude were taken to be $10 \mathrm{~mm}$ and $114 \mathrm{MPa}$, respectively. A significance level of $5 \%$ was used to construct $90 \%$ confidence intervals, and 500 particles were used. The results of particle filtering prognostics are shown in Figure 11.

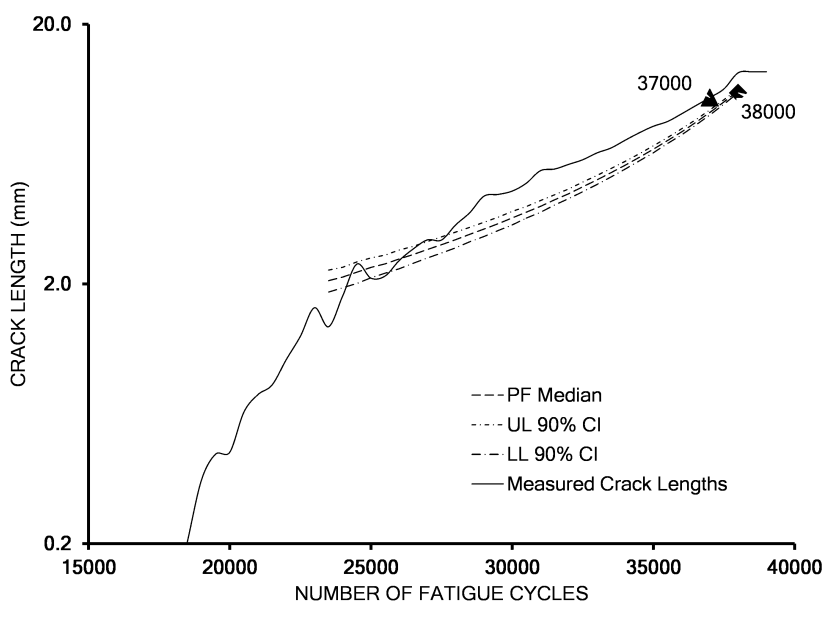

Figure 11. Crack length estimates using Particle Filtering.

For this coupon, the $10 \mathrm{~mm}$ crack length was reached at 37,000 cycles, which matches well with the median prediction of 38,000 cycles; however, these results are very unstable depending on the material properties $(C, m, a)$ statistical distributions input and PF Bayesian model updating, including random initializations. The estimates of crack lengths are consistently biased to shorter lengths than the corresponding actual experimental measurements. The PF-based prognostics technique was demonstrated to work well with off-line recorded fatigue crack length measurements, for selected number of coupons. The margin of error was initially significantly larger than the NARX technique; however, the $\mathrm{PF}$ technique resulted in diminishingly smaller errors as the updating progressed to the terminal threshold crack length, the end of fatigue life.

The material properties (C, m) and crack length (a) statistical distribution parameters may not always be applicable to live real-time monitoring and fatigue prognostics crack initiation and growth. It is desirable to conduct such trials to further enhance these PF and other hybrid prognostics techniques.

These results demonstrate the need for hybrid techniques combining specific customized crack growth prior results obtained from databased machine learning methods like NARX approach with Bayesian updating particle filtering methods. It is desirable to test these techniques for live realtime implementation of fatigue crack prognostics.

\section{Conclusions and Future Plans}

A structural health monitoring system was shown to be sensitive to crack growth in aluminum 7075-T6 coupons subject to fatigue loading. AFGROW and NASGRO simulations of crack growth in the same material were shown to be accurate for crack sizes greater than $5 \mathrm{~mm}$. The NARX technique was shown to accurately simulate crack sizes longer than $5 \mathrm{~mm}$ through non-linear neural network based 
machine learning. Experimentally recorded off-line crack growth results were combined with statistical material properties using particle filtering to conduct crack growth prognostics; initial results demonstrated the applicability of PF technique to off-line recorded fatigue crack growth.

Future work will incorporate the combination of NARX technique with the particle filtering to accomplish accurate crack growth prognostics, in live real-time setting. Subsequently, the structural health monitoring data will be incorporated into the particle filtering technique to provide accurate estimates and prognostics of individual fatigue test application.

\section{ACKNOWLEDGEMENT}

The authors would like to acknowledge partial support from NSF Award OIA-154079.

\section{REFERENCES}

An, D., Choi, J.-H., \& Kim, N. (2013). Prognostics 101: A tutorial for particle filter-based prognostics algorithm using Matlab. Reliability Engineering and Systems Safety. 115, 161-169.

Haile, M., Chen, T.-K., Sediles, F., Shiao, M., \& Le, D., (2012). Estimating crack growth in rotorcraft structures subjected to mission load spectrum. International Journal of Fatigue, vol. 43, pp. 142-149.

Iglesias, E. E., Haynes, R. A., \& Shiao, C. Y., "Inspection Correlation Study of Ultrasonic-Based In Situ Structural Health Monitoring Monthly Report for December 2014January 2015,” ARL-TN-0671, 2015.

Johnson, W.S., (2010). The History, Logic and Uses of the Equivalent Initial Flaw Size Approach to Total Fatigue
Life Prediction. Procedia Engineering, vol. 2(1), pp. 4758.

NASGRO, (2008). Fracture Mechanics and Fatigue Crack Growth Analysis Software, Version5.2. NASA-JSC and Southwest Research Institute.

Newman, J.C. \& Ramakrishnan, R., (2016). Fatigue and crack-growth analyses of riveted lap-joints in a retired aircraft. Int. J. Fatigue. vol. 82, pp. 342-349.

Paris, P, \& Erdogan F. A, (1963). Critical analysis of crack propagation laws. J Basic Eng. vol. 85.

Siegelmann, H., Horne, B., \& Giles, C. (1997). Computational Capabilities of Recurrent NARX Neural Networks. IEEE Transactions on Systems, Man,and Cybernetics-Part B: Cybernetics, 27 (2), 208-215.

Vasudevan, A.K., Sadananda, K., \& Iyyer, N. (2016). Fatigue damage analysis: Issues and challenges. Int. J. Fatigue, vol. 82, 120-133.

Vehorn, K. A., DeSimio, M. P., Olson, S. E., Brown, K. S., \& Leonard, M. S., "Stability of Guided Wave Signals from Bonded Piezoelectric Sensors,” SPIE Proceedings Vol. 8695: Health Monitoring of Structural and Biological Systems 2013, San Diego, CA, March 11-14, 2013.

Weiss, V. \& Ghoshal A. (2014). On the search for optimal damage precursors. Structural Health Monitoring. 13(6), 601-608.

Zio, E. and Peloni, G. (2010). Particle Filtering Prognostics Estimation of the Remaining Useful Life of Nonlinear Components. Reliability Engineering \& System Safety 96(3), 403-409. 